\title{
Еlección de Destinos Turísticos: influencia del grado de implicación y conocimientos previos de turistas ${ }^{1}$
}

\begin{abstract}
Raúl Valdez, M.?
RESUMEN: Los modelos de comportamiento del consumidor sugieren que la percepción, las imágenes, las actitudes, el nivel de conocimiento previo y el grado de implicancia delos viajerosinfluyen demanera determinante sobre la composición interna de los conjuntos de elección de los turistas. Este estudio busca confirmar la estabilidad en la composición de los siguientes conjuntos: evocado, inerte y de rechazo; también busca verificar la influencia que sobre la composición de dichos conjuntos ejercen de manera particular el nivel de conocimientos previos y el grado de implicación que en materia de viajes de placer poseen los turistas. Los resultados demuestran que los conjuntos evocado y de rechazo, cambian su composición interna de manera constante y el nivel de conocimientos y el grado de implicancia de los turistas, además de ser variables no correlacionadas, no ejercen una influencia significativa sobre la composición de los conjuntos de elección de los viajeros.
\end{abstract}

PALABRAS CLAVE: marketing turístico, comportamiento del consumidor, conocimiento previo e implicancia del turista, formación de conjuntos, elección de los destinos turísticos.

ABSTRACT: The behaviormodels of the customer suggest that the perception, the images, the attitudes, the tourists' previous level of knowledge and commitment influence deeply in the collection of preferences elected by them. This study seeks for confirming the stability in the composition of the following groups: evocative, inert and disposable; also verifies the influence that the tourists'

1. Trabajo presentado en el $3^{\circ}$ Congreso Latinoamericano de Investigación Turística "Conocimiento: puente hacia la integración y excelencia turística", Universidad Austral de Chile, Valdivia, octubre-noviembre del 2001.

‘́. Ph.D. Departamento de Administración de Hoteles y Restaurantes. Universidad de las Américas, Puebla. México. E-mail:rvalde : imail.udlap.mx 
previous level and commitment degree cause, in a particular way, in the composition of these groups, in leisure tours. The results demonstrate that the groups evocative and disposable change their internal composition constantly and that the tourists' knowledge level commitment degree besides being variable and not correlated does not cause a significant influence in the composition of elected group of tourists.

KEYWORDS: touristic marketing, customer behavior previous knowledge, tourist commitment, groups composition, tourist destination election.

\section{Introducción}

En el dominio conceptual del marketing, la evolución de los campos de investigación sobre el comportamiento del consumidor ha dado lugar a múltiples áreas de interés a lo largo de su historia. En los años cincuenta, los esfuerzos de los investigadores se enfocaron principalmente hacia los determinantes sociales del comportamiento. En la década de los sesenta, el interés se volcó al desarrollo de modelos explicativos del comportamiento, y, posteriormente, en épocas siguientes, los estudios de investigación pusieron énfasis en la influencia de las diferencias culturales, sobre el papel de los ritos, la fantasía y el simbolismo; finalmente, los estudios volcaron su interés sobre el efecto que ejerce la relación actitudcomportamiento en la decisión de compra de los consumidores.

En el dominio conceptual del marketing de servicios, los resultados de diversas investigaciones empíricas han propiciado el abandono del paradigma tradicional del intercambio que consideraba este fenómeno como un simple mecanismo unidireccional del tipo estímulo-respuesta. Desde una nuevaperspectiva, los investigadores han logrado construir el nuevo paradigma de la "dualidad del intercambio" (dyadic exchange paradigm) según el cual, la relación vendedorcomprador ya no puede ser considerada como una simple secuencia de causa a efecto, sino más bien, como una relación transaccional en la que el comprador, involucrado fuertemente en los procesos de negociación y de equilibrio del poder, asume un papel activo y vuelve más compleja la relación de intercambio.

Este nuevo paradigma de dualidad respecto de la naturaleza de las relaciones entre vendedores y compradores de servicios reviste relevancia en el campo del turismo; esto se debe a que el grado de involucramiento del consumidor representa un factor clave tanto del proceso de servucción como dentro de las fases del proceso de consumo.
En el campoespecífico del marketing turístico, la mayoría de los modelos de comportamiento del consumidor. sugieren que existen relaciones interactivas entre variables tales como la percepción, las imágenes, las actitudes, las experiencias acumuladas y el grado de implicancia de los viajeros, y que éstas influyen de manera determinante en el proceso de elección de los destinos turísticos.

\section{Elección en el Proceso de Decisión de los Destinos Turisticos}

\section{Importancia de los Conjuntos de Elección}

En el contexto de los modelos descriptivos del comportamiento del turista, diversos constructos como el conjunto evocado (Narayana y Markin, 1975; Woodside y Lysonski, 1989; Um y Crompton, 1990; Crompton, 1992), la estructura de preferencia (Moutinho, 1987), las asociaciones afectivas hacia los destinos preferidos (Woodside y Lysonski, 1989) y la imagen asociada a los destinos de viaje (Schmoll, i977; Mathieson y Wall, 1982) constituyen los fundamentos conceptuales de la elección de los destinos turísticos.

Tomando como referncia la noción que los autores antes mencionados denominan "psicologíade simplificación del consumidor"' (consumer's psychology of simplification), Narayana y Markin (1975) sostienen que los consumidores buscan siempre reducir su conjunto total de opciones de compra cuando deben hacer una elección entre muchos productos o marcas.

Mediante un estudio exploratorio estos autores demostraron que los consumidores identifican y seleccionan los bienes o servicios (o sus marcas comerciales) que consideran como posibilidades de compra y los clasifican en su mente formando grupos conocidos como "conjuntos de elección" (choice sets). Estos conjuntos de elección se clasifican a su vez en tres tipos principales:

- el denominado conjunto "evocado" (evoked set), en el cual, el consumidor clasifica los bienes o servicios que conoce, prefiere y que está dispuesto a comprar como primera opción.

- el segundo tipo corresponde al conjunto "inerte" (inert set), dentro del cual, el consumidor agrupa los bienes o servicios que conoce de manera limitada y que podría considerar como substitutos de los bienes "evocados".

- finalmente, en el conjunto denominado "de rechazo" (inept set), el consumidor clasifica los bienes o servicios que no desea adquirir o que incluso trata de evitar como opciones de compra.

Examinando las relaciones existentes entre los diferentes niveles de conocimiento de los consumidores y sus preferencias personales hacia los destinos de viaje, Woodside y Carr (1988) consideran que: 
En la vida real, mientras los consumidores asocian un beneficio particular con cada destino, están de acuerdo con substituir una combinación benefício-destino por la concurrencia entre una unidad beneficio-destino, dependiendo delvalorpercibidoen cada alternativa y en la decisión aplicada al realizar las elecciones.

En este mismo contexto, Woodside y Sherrell (1977) han realizado una investigación empírica sobre una muestra de 71 viajeros; los resultados demostraron que el conjunto evocado de los turistas está integrado por cuatro destinos como máximo y que, las actitudes adoptadas por el viajero hacia estos destinos evocados son mucho más positivas que las actitudes detectadas con respecto a los destinos que integran los conjuntos inerte y de rechazo.

Siguiendo esta misma corriente de opinión, el estudio de Crompton y Ankomah (1993) sugiere que los viajeros forman un conjunto "preliminar" de destinos posibles (early consideration set) a partir del cual, depurando sus percepciones, integran un conjunto "ulterior" (late consideration set) y realizan su elección a partir de éste último. Los autores establecen textualmente:

Los criterios usados para evaluar las alternativas en un conjunto "preliminar" de destinos en principio focalizaránen cualidades relativas a los atributos de destino, mientras que, los criterios utilizados para evaluar las alternativas en un conjunto "ulterior" en principio localizarán en las limitaciones asociados con cada uno de los destinos posibles.

Un aspectoque merece mayor grado de reflexión es el hecho de que, pasando inadvertido el carácter dinámico de las percepciones, motivaciones y actitudes, prácticamente todos los modelos explicativos del comportamiento de los consumidores consideran que la composición interna de los conjuntos evocado, inerte y de rechazo permanece estable a lo largo de periodos de tiempo más o menos prolongados.

\section{Grado de Implicancia y Conocimientos Previos de Viajeros}

Si bien la percepción constituye el mecanismo interpretativo de base de todos los estímulos percibidos por el individuo, solamente algunas investigaciones empíricas identifican los atractivos naturales de los destinos turísticos (Crompton, 1979; Saleh y Ryan, 1992), la interpretación emotiva de las características físicas de los sitios (Pigram y Dunn, 1976), el grado de implicancia o de involucramiento de los viajeros (Celci y Olson, 1988; (Dimanche, et al., 1991; Reid y Crompton, 1993; Schuett, 1993; Havitz y Dimanche, 1990; citados por Jamrozy et al., 1996) y el nivel de los conocimientos previos del viajero, comoestímulos fundamentales de la interpretación perceptual que el turista utiliza en la formación de sus conjuntos de elección.

Los conocimientos que acumulan los consumidores a través del tiempo, respectos de los productos o servicios, constituyen un factor importante de su marco de referencia motivacional y por ende, de la formación de actitudes al momento de elegir un producto determinado (Bagozzi et al. (1991, citados por Schiffman y Kanuk, 1997). En el campo del turismo, la literatura sugiere que los conocimientos previos adquiridos por los viajeros en materia de viajes de placer se constituyen en un antecedente importante en el momento de elegir los destinos turísticos. En este sentido, los resultados de diversos estudios hacen suponer que un elevado conocimiento en materia de viajes otorga al individuo una mayor habilidad para modificar constantemente sus juicios de evaluación sobre los diferentes destinos turísticos que puede elegir en un momento dado (Pigram y Dunn, 1976; Milman y Pizam, 1995).

El grado de implicancia (involvement) del individuo constituye otro factor que complen $r_{1}$ enta sus conocimientos previos contribuyendo también a delinear la representación mental que el turista asocia a los sitios de diversión. En un sentido amplio, Rothschild (1984, citado por Jamrozy et al., 1996) define la implicancia (involvement) en los siguientes términos:

Un estado de motivación, estímulo o interés. Este estado se evidencia en un proceso. Es acarreado porvariables externas recientes (la situación, el producto, lascomunicaciones) y por variables pasadas (permanencia, y por variables centrales). Estas consecuencias son tipos de elecciones, acciones y decisiones.

Por su parte, en el contexto específico del turismo, Havitz y Dimanche (1990, citados por Jamrozy et al., 1996) definen la implicación del individuo en el campo de las actividades turísticas de la siguiente manera:

La inplicancia es un estado de motivación psico social, estimulo o interés entre actividade individuales y de recreación, destinos turisticos o elementos asociados a uno de los puntos en cuestión, caracterizado por la percepción de los siguientes elementos: importancia, valor placer, valor signo, probabilidad de riesgo y consecuencias de los riesugs

En resumen, podría establecerse que, en cuanto a la relación existente entre el grado de implicancia hacia los viajes turísticos y la composición de los conjuntos de elección del viajero, es de esperarse que los individuos que poseen un alto grado de implicancia hacia los viajes de placer sean más propensos a modificar sus conjuntos de elección que aquellos que se consideran menos involucrados en estas actividades.

Como podemos observar en el campo del turismo, la mayoría de los modelos de comportamiento del consumidor sugieren que el viajero elige los destinos de diversión partiendo de los sitios de atracción turística que integran sus conjuntos de 
elección, los cuales, según los resultados de investigaciones empíricas, permanecen estables durante largos periodos de tiempo. Si bien se partimos de la base de entender que los consumidores en general y los turistas en particular están constantemente expuestos a la influencia de factores dinámicos pertenecientes a los entornos económicos, sociales, culturales y tecnológicos (Pearce y Caltabiano, 1983; Unger y Kernan, 1983; Manell e Iso-Ahola, 1987; Mazursky, 1989; Ryan, 1991; Otto y Ritchie, 1996; Laplante, 1996), la suposición de que los destinos turísticos que integran los conjuntos de elección permanecen estables en la mente de los viajeros, es una tesis discutible.

\section{Metodología y Resultados de un Estudio}

\section{Naturaleza y Objetivos}

Los objetivo centrales de este estudio son:

- confirmar la naturaleza estable de la composición interna de los conjuntos de elección del turista;

- verificar la influencia que ejerce el grado de implicancia del turista sobre la composición de sus conjuntos de elección;

- verificar la influencia que ejerce el nivel de conocimientos previos que tiene el turista respectos de viajes de placer, sobre la composición de sus conjuntos de elección;

- identificar si existe una correlación significativa entre el grado de implicancia y el nivel de conocimientos previos que el turista tiene sobre los viajes de placer que pueda influir en la composición de sus conjuntos de elección.

Elpresente estudio se llevó a cabo en la provincia de Québec, Canadá; se trata de una investigación descriptiva y longitudinal. Se tomó como población objetivo a individuos de ambos sexos con experiencia turística en viajes dentro del territorio canadiense o en otros países. Una vez determinado el marco muestral, se diseñaron los instrumentos de medición necesarios y se aplicaron en tres ocasiones diferentes en un periodo total de 110 días con un intervalo de 30 días entre una aplicación y otra.

\section{Hipótesis de Investigación}

Las iipótesis de investigación del estudio son:

- $\mathrm{H}_{0}$ : los destinos turísticos de los conjuntos evocado, inerte y de rechazo, no cambian en el corto plazo;
- $\mathrm{H}_{1}$ : los destinos turísticos de los conjuntos evocado, inerte y de rechazo, cambian en el corto plazo;

- $\mathrm{H} 2$ : el grado de implicancia del turista no influye de manera significativa sobre la composición de sus conjuntos evocado, inerte y de rechazo;

- H2 : el grado de implicancia del turista influye de manera significativa sobre la composición de sus conjuntos evocado, inerte y de rechazo;

- $3_{0}$ : el rivel de conocimientos previos que el turista tiene sobre viajes de placer, no influye de manera significativa sobre la composición de sus conjuntos evocado, inerte y de rechazo;

- H3, el nivel de conocimientos previos que el turista tiene sobre viajes de placer, influye de manera significativa sobre la composición de sus conjuntos evocado, inerte y de rechazo;

- $\mathrm{H}_{4}$ : no existe una correlación significativa entre cl grado de implicancia y el nivel de conocimientos previos que tiene el turista respecto de viajes de placer;

- $\mathrm{H}_{4}$ : existe una correlación significativa entre el grado de implicancia y el nivel de conocimientos previos que tiene turista respecto de viajes de placer.

\section{Instrumentos de Medición, Muestra y Recolección de Datos}

Para la recolección de la información necesaria se utilizaron cuestionarios integrados por preguntas abiertas, preguntas de opción múltiple y escalas de medición especialmente diseñadas para los fines del estudio.

Para determinar la composición de los conjuntos evocado, inerte y de rechazo, se utilizaron preguntas abiertas inspiradas en los instrumentos utilizados por Woodside y Sherrell (1977) y por Woodside y Lysonski (1989).

Para determinar el nivel de conocimientos previos que el turista tiene en materia de viajes de placer, se construyó una escala tipo Likert compuesta por cuatro enunciados y siete opciones de respuestas posibles. En su diseño, se utilizó como base la escala desarrollada por Engel, Blackwell y Miniard (1993) para identificar el nivel de familiaridad de los individuos con relación a un objeto dado. Por tratarse de una escala aditiva, la medida de los conocimientos previos del turista es calculada a partir de la suma de los valores asignados a las respuestas de los enunciados de la escala. De esta forma, las puntuaciones altas ( 20 a 28 puntos) se corresponden con un nivel alto de conocimientos previos sobre viajes turísticos, mientras que las puntuaciones bajas ( 4 a 12 puntos) se refieren a la escasez de dichos conocimientos.

Para identificar el grado de implicancia de los viajeros con respecto a los viajes de turismo, se elaboró otra escala de tipo Likert; ésta contenía 16 enunciados con siete opciones posibles de respuesta. El total de enunciados se agrupó en cuatro factores que miden de manera específica el simbolismo concedido a la experiencia de viaje ( 3 enunciados), la autoestima del viajero ( 5 enunciados), la probabilidad de haber hecho una mala elección (4 enunciados) y el riesgo asociado a la elección del 
destino (4 enunciados). Por tratarde también de una escala aditiva, las puntuaciones altas ( 80 a 112 puntos) denotaban un alto grado de implicancia del viajero, mientras que los puntajes bajos (16 a 48 puntos), correspondían a bajos grados de involucramiento en materia de viajes de placer. Esta escala es una adaptación que se realizó para los fines de este estudio a partir de un instrumento denominado CIP (Consumer Involvement Profiles) desarrollada por Laurent y Kapferer (1985, citados por Bearden, Netemeyer y Mobley, 1993, con el fin de determinar el nivel global de implicancia de los individuos frente a los productos o servicios considerados como opciones viables de compra.

Se validó la confiabilidad de las escalas utilizadas mediante el índice Alpha $(\alpha)$ de Cronbach. Los resultados obtenidos fueron satisfactorios al mostrar valores globales de $\alpha=0.8692$ para el caso de la escala que mide los conocimientos previos, y de $\alpha=0.9207$ (simbolismo del viaje), $\alpha=0.7275$ (autoestima), $\alpha=0.7850$ (probabilidad de mala elección) y de $\alpha=0.6689$ (riesgo asociado a la elección), para los factores quemiden globalmente el grado de implicancia de los viajeros en materia de viajes turísticos.

A partir de la población considerada como universo de estudio, se determinó como marco muestral el conjunto total de estudiantes inscriptos en los programas de Maestría en Administración de Empresa (MBA) y de Maestría en Gestión de Proyectos (MGP) de la Universidad de Québec en Montréal, Canadá.

A partir de allí, se utilizó una muestra de conveniencia formada inicialmente por 153 individuos, de los cuales, solo 103 individuos formaron parte de la muestra final para el análisis, debido a razones inherentes a la naturaleza longitudinal del estudio (efecto de mortalidad). Tomando en cuenta que con muestras de al menos

100 individuos se puede asegurar un mínimo de precisión en los resultados de los análisis para efectos de inferencia estadística (Perrien, Chéron y Zins, 1984), se consideró que una muestra de 103 individuos cumplía con este requisito.

\section{Análisis de Datos}

Para probar la hipótesis 1 sobre el dinamismo de los conjuntos de elección del turista, se utilizó el test de Cochran; éste es un método no paramétrico que se emplea para probar si dos o más muestras relacionadas muestran proporciones o frecuencias estadísticamente diferentes(Perrien, Chéron y Zins, 1984). Lautilización de este testse justificó debido a que el objetivo del análisis era verificar si los destinos turísticos clasificados en los conjuntos evocado, inerte y de rechazo, continuaban siendo los mismos durante las tres mediciones del estudio.

Para realizar el análisis de caso hubo que transformar los datos categóricos (destinos turísticos), en datos dicotómicos; éstos representan frecuencias en una tabla de $n$ renglones y c columnas de manera que la hipótesis nula establece que las frecuencias de los datos es la misma en cada medición efectuada y representada por cada columna. La regla de decisión fue: rechazar $\mathrm{H}_{0}$ si el nivel de significancia (p) $<\alpha$, donde $\alpha=0.05$.
Para probar las hipótesis 2, 3 y 4 que se refieren a las influencias del grado de implicancia del nivel de conocimientos previos del turista en materia de viajes de placer y de la posible correlación existente entre estas dos variables sobre la formación de susconjuntos de elección, se utilizó un análisis de varianza multivariado (MANOVA) con medidas repetidas.

El empleo del análisis de varianza multivariado (MANOVA) se justifica debido a que este método permite evaluar la equivalencia de los valores medios (vectores) de las variables dependientes entre los diferentes grupos considerados, medidos en ocasiones sucesivas. Para este caso particular, el estudio busca identificar la influencia que, de manera simultánea, ejercen tanto el grado de implicancia como el nivel de conocimientos previos de los viajeros respecto de viajes turísticos a lo largo de las tres mediciones realizadas (Hair Jr. et al., 1995).

En el contexto del análisis, el factor "intra" (Within-Subjects Factor) está representado por la existencia o inexistencia de cambios en la composición de los conjuntos evocado, inerte y de rechazo a lolargo de las tres mediciones del estudio; mientras que el factor "inter" (Between-Subjects Factor) está representado por el nivel de conocimientos y el grado de implicancia del turista en materia de viajes de placer. De esta forma, el análisis busca descubrir si el nivel de conocimientos y el grado de implicancia pueden explicar las variaciones observadas en la clasificación de los destinos turísticos dentro de cada uno de los conjuntos de elección de los entrevistados. La regla de decisión fue: rechazar $\mathrm{H}_{0}$ si el nivel de significancia (p) $<\alpha$, donde $\alpha=0.05$.

\section{Resultados}

Los resultados de la presente investigación demostraron que la composición interna de los conjuntos de elección de los turistas no es del todo estable. Los resultados del test de Cochran (Tabla 1 ) indican que la composición de los conjuntos evocado y de rechazo, cambian aún en el corto plazo $\left(\mathrm{Q}_{\text {cvocado }}=0.0179<0.05 \mathrm{y}\right.$ $\mathrm{Q}_{\text {rechazo }}=0.0278<0.05$ respectivamente). Sin embargo, el conjunto inerte no parece demostrar cambios significativos a través del tiempo. $\left(Q_{\text {incre }}=0.6428>0.05\right)$.

TABLA 1 - RESULTADOS DEL TEST DE COCHRAN

\begin{tabular}{l|c|l|l|l}
\hline \multicolumn{1}{c|}{ Test } & $\boldsymbol{n}$ & Estadistico & g. I. & Significancia \\
\hline Cochran (evocado) & 103 & $\mathbf{Q}=8,0476$ & 2 & 0,0179 \\
Cochran (inerte) & 103 & $\mathbf{Q}=0,8837$ & 2 & 0,6428 \\
Cochran (de rechazo) & 103 & $\mathbf{Q}=7,1667$ & 2 & 0,0278 \\
\hline
\end{tabular}


Los resultados llevan a rechazar parcialmente la hipótesis $\mathrm{H} 1_{0}$ dado que ésto sóo es válido para el caso de los conjuntos evocado y de rechazo, y por consiguiente se debe aceptar $\mathrm{H} 1_{0}$ sólo para el caso del conjunto inerte.

Para el caso del la hipótesis 2, referida al grado de implicancia, se confirmó que esta variable no ejerce una influencia determinante sobre las variaciones que sufre la composición de los conjuntos de elección del turista. $\left(\mathrm{F}_{\text {cvocado }}=0.512>0.05\right.$,

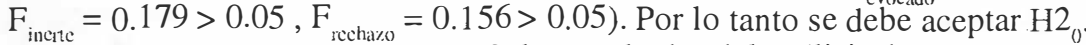

Para el caso de la hipótesis 3 , los resultados del análisis demostraron que el nivel de conocimientos del turista en materia de viajes de placer, no ejerce una influencia significativa sobre la composición de sus conjuntos evocado, inerte y de

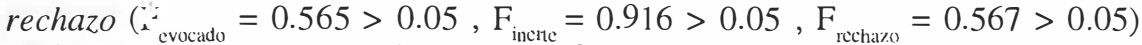
(Tabla 2). Por lo tanto se debe aceptar $\mathrm{H}_{0}$.

Finalmente tampoco logró encontrarse una correlación relevante entre el nivel de conocimientos y el grado de implicancia de los turistas con relación a viajes de placer $\left(\mathrm{F}_{\text {evociado }}=0.964>0.05, \mathrm{~F}_{\text {incite }}=0.693>0.05, \mathrm{~F}_{\text {recharo }}=0.365>0.05\right)($ Tabla 2). Por lo tanto se debe aceptar la hipótesis $\mathrm{H}_{0}$.

\section{Conclusiones}

Citase a continuación las conclusiones del estudio:

- Los conjuntos evocado y de rechazo de los turistas cambian constantemente de composición; esto significa que los destinos turísticos preferidos por un viajero en un momento dado, así como los no deseados, no serán exactamente los mismos en un periodo de tiempo relativamente corto. Esta conclusión permite suponer que tanto los destinos turísticos preferidos, como aquellos no deseados por el viajero en un momento determinado, son constantemente reclasificados en la mente del turista; por ello podría pensarse que su elección o su rechazo se debería más a una decisión in pulsiva que planeada. Por loconsiguiente, y en contraposición a lo que la literatura generalmente establece, los conjuntos de elección del turista parecen cambiar su composición interna de manera constante.

- Tanto el grado de implicancia de los viajeros como los conocimientos previos que posean en materia de turismo, no son factores que contribuyan a modificar significativamente su preferencia, su indiferencia o incluso su rechazo por los destinos turísticos. Con base en esto, la suposición de que tanto el nivel de conocimiento que los turistas poseen en materia de viajes de placer como su grado de implicancia en los viajes que realizan son factores que contribuyen a preferir o a rechazar determinados destinos turísticos parece ser una tesis no completamente cierta.

- En ese mismo contexto, a falta de correlación significativa entre el grado de implicancia de los turistas y su nivel de conocimientos previos en materia de viajes de placer parece indicar que estos factores actúan de manera independiente en los procesos de clasificar y posteriormente de decidir los destinos turísticos que serán visitados. Por lo tanto, esta conclusión sugiere que si bien estas variables pueden influir en la percepción que el turista tiene de los destinos de viaje, no se puede asegurar de manera definitiva que ejerzan una influencia conjunta y significativa en la manera en que los viajeros clasifican los destinos turísticos dentro de cada uno de sus conjuntos evocado, inerte y de rechazo.

TABLA 2

\begin{tabular}{|c|c|c|c|c|c|}
\hline Fuente de variación & $\begin{array}{l}\text { Suma de } \\
\text { cuadrados }\end{array}$ & gl. & $\begin{array}{l}\text { Media de } \\
\text { los } \\
\text { cuadrados }\end{array}$ & F & Signit.t \\
\hline \multicolumn{6}{|c|}{ Test de los efectos "intra" (Within-Subjects Effects) } \\
\hline $\begin{array}{l}\text { Tiempo entre aplicacione } \\
\text { Cambios de: }\end{array}$ & & & & & \\
\hline a) destinos evocados & 0,19600 & 1 & 0,19600 & 1,056 & 0,307 \\
\hline b) destinos inertes & 0,02411 & 1 & 0,02411 & 0,185 & 0,668 \\
\hline c) destinos rechazados & 0,12800 & 1 & 0,12800 & 0,883 & 0,350 \\
\hline
\end{tabular}

Test de los efectos " inter" (Between-Subjects Effects)

\begin{tabular}{|c|c|c|c|c|c|}
\hline \multicolumn{6}{|l|}{ Grado de implicación: } \\
\hline b) destinos inertes & 0,460 & 1 & 0,460 & 1,834 & 0,179 \\
\hline c) destinos rechazados & 0,670 & 1 & 0,670 & 2,045 & 0,156 \\
\hline \multicolumn{6}{|l|}{ Nivel de conocimientos: } \\
\hline a) destinos evocados & 0,37300 & 2 & 0,18600 & 0,574 & 0,565 \\
\hline b) destinos inertes & 0,04385 & 2 & 0,02192 & 0,087 & 0,916 \\
\hline c) destinos rechazados & 0,37400 & 2 & 0,18700 & 0,571 & 0,567 \\
\hline \multicolumn{6}{|c|}{ Interacción } \\
\hline \multicolumn{6}{|l|}{$\begin{array}{l}\text { Cambios de destino con } \\
\text { grado de implicación: }\end{array}$} \\
\hline a) destinos evocados & 0,02927 & 1 & 0,02927 & 0,158 & 0,692 \\
\hline b) destinos inertes & 0,50100 & 1 & 0,50100 & 3,842 & 0,053 \\
\hline c) destinos rechazados & 0,09990 & 1 & 0,09990 & 0,688 & 0,409 \\
\hline \multicolumn{6}{|l|}{$\begin{array}{l}\text { Grado de implicación y } \\
\text { rivel de conocimientos: }\end{array}$} \\
\hline a) destinos evocados & 0,01370 & 2 & 0,00685 & 0,037 & 0,964 \\
\hline b) otsinos inertes & 0,09624 & 2 & 0,04812 & 0,369 & 0,693 \\
\hline c) destinos rechazados & 0,296 & 2 & 0,148 & 1,020 & 0,365 \\
\hline
\end{tabular}

* Dos mediciones efectuadas : primera medición $=$ resultados entre la $1_{\mathrm{a}}$ y 2 aplicación; segunda medición $=$ resultados entre la 2 y la 3 aplicación.

† Condición de esfericidad satisfecha. 


\section{Bibliografía}

BEARDEN, W. O.; NETEMEYER R. G.; MOBLEY. M. F. 1993. Handbook of marketing scales: multi-item measures for marketing and consumer behavior research. Newbury Park, Cal. USA: Sage Publications.

CELSI, R. L.; OLSON. J. C. 1988. The role of involvement in attention and comprehension processes. Journal of Consumer Research, n. 15, sep., p. 210-224.

CROMPTON '. 1. 1979. An assessment of the image of Mexico as a vacation destination and the influence of geographical location upon that image. Journal of Travel Research, spring, p. 18-23.

CROMPTON, J. L. 1992. Structure of vacation destination choice sets. Annals of Tourism Research, Menemonie, vol. 19. p. 420-434.

CROMPTON, J. L. y P. K. Ankomah. 1993. Choice sets propositions in destination decisions. Annuls of Tourism Research, Menemonie, v. 20, p. 461-476.

ENGEL, J. F.; BLACKWELL, R. D.; MINIARD. P. W. 1993. Consumerbehavior, 7ed, Orlando, FI. USA : TheDryden, $846 \mathrm{p}$.

HAIR JR., J. F.; ANDERSON, R. E.; TATHAM, R. L.; BLACK, W. C. 1995. Multivariate data anal sis with readings. 4. ed. New Jersey, USA : Prentice-Hall.

JARROZY, U.; BACKMAN, S. J.; BACKMAN, K. F. 1996. Involvement and opinion leadership in tourism. Annals of Tourism Research, Menemonie, vol.23, n. 4, p. 908-924.

LAPLANTE, M. 1996. L'expérience touristique contemporaine: fondements sociaux y' culturels. Sainte-Foi, Québec: Université du Québec

MANNELL, R. C.; ISO-AHOLA, S. E. 1987. Psychological nature of leisureand tourism experience. Annals of Tourism Research, Menemonie, v. 14, p.314-331.

MATHIESON, A.; WALL, G. 1982. Tourism : economic.physic and social impacts. U.K : Longman.

MAZURSKY, D. 1989. Past experience and future tourismdecisions, Annals of Tourism Research, Menemonie, v. 16, p. 333-344.

MILMAN, A.; PIZAM, A. 1995. Therole of awareness and familiarity with a destination: the Central Florida case. Journal of Travel Research, winter, p.21-27.

MOUTINHO, L. 1987. Consumer Behavior in Tourism. European Journal of Markying, v. 21, n. 10, pp. 5-44.

NARAYANA, C. L.; Markin, R. J. 1975. Consumer behavior and productperformance: analternative conceptualization. Journal of Marketing, vl. 39, oct., p. 1-6.

OTTO, J. E.; Ritchie, J. R. B. 1996. The service experience in tourism. Tourism Management, London, v. 17, n. 3, p. $165-174$.

PEARCE, P. L.; CALTABIANO, M. L. 1983. Inferring travel motivation from traveler's experiences, Journal of Travel Research, USA, fall, pp. 16-20.

PERRIEN, C Z ZINS. 1984. Recherche en markining: méthodes y clécisions. Boucherville, Québec, Canada: Gaëtan Morin.

PIGRAM, J. J.; DUNN. J. B. 1976. Monitoring recreation behaviour. Journal of Travel Research, USA, vl. 15, p. 14-18.

RYAN, C. 1991. Recreational tourism: a social science perspective. London \& New York: Routledge.

SALEH, F.; RYAN, C. 1992. Client perceptions of hotels: a multi-attribute approach. Tourism Management, London, june, p. 163-168.

SCHIFFMAN, L. G.; KANUK, L. L., 1997. Comportamiento del consumidor. 5. ed., México: Prentice Hall Hispanoamericana.

SCHMOLL, G. A. 1977. Tourism promotion. London: Tourism International.

UM, S.; CROMPTON, J. L. 1990. Attitude determinants in tourism destination choice. Annals of Tourism Research, Menemonie, v. 17, p. 432-448.

UNGER, L. S.; KERNAN, J. B. 1983. On the meaningof leisure: an investigation of some dyerminants of the subjective experience. Journal of Consumer Research, v. 9, march, p. 381-392.

WOODSIDE, A. G.; SHERRELL, D. 1977. Traveler evoked, inept, and inert sets of vacation destination. Journal of Travel Research, USA, v. 16, winter, pp. 14-18.

ivOODSIDE, A. G.; CARR, J. A. 1988. Consumer decision making and competitive marketing strategies: applications for tourism planning. Journal of Travel Research, USA, winter, p.2-7.

WOODSIDE, A. G.; LYSONSKY, S. 1989. A general model of travelerdestination choice. Journal of Travel Research, USA, spring, p. 8-14.

\section{Recibido en $5 / 11 / 02$}

Aprobado en 15/11/02 